\title{
Improved Stereo Image Dehazing Approach
}

\author{
${ }^{1}$ Yu-Chen Wu, ${ }^{2}$ Chin-Chen Chang, ${ }^{3}$ Der-Lor Way, ${ }^{4}$ Zen-Chung Shih \\ ${ }^{1,4}$ Institute of Multimedia Engineering, National Chiao Tung University, Hsinchu, Taiwan; \\ ${ }^{2}$ Department of Computer Science and Information Engineering, National United University, Miaoli \\ Taiwan; \\ ${ }^{3}$ Department of NewMedia Art, Taipei National University of Arts, Taipei, Taiwan; \\ ccchang@nuu.edu.tw
}

\begin{abstract}
Numerous image dehazing algorithms have been studied intensively. However, most dehazing algorithms operate on single images. These algorithms produce inconsistent results if they are used to dehaze stereo images iteratively. In this paper, we present a novel dehazing approach for stereo images based on cross bilateral filtering. In this approach, we simultaneously estimate scene depth and dehaze the stereo images. The proposed approach is based on the observation of depth cues in the stereo images. Depth cues are mainly used to avoid inconsistent results, and the cross bilateral filter is used to preserve shape details. The results demonstrate that the proposed approach can deliver superior results to those of previously published methods.
\end{abstract}

Keywords: Dehazing; Stereo Images; Image Restoration; Depth Estimation.

\section{Introduction}

If a scene is photographed under inclement weather conditions, such as fog or haze, the quality of the captured images would be degraded. This is not a direct process of degradation; the light is attenuated as it moves toward the camera. The light entering the camera is blended with ambient light reflected from the atmospheric particles. Therefore, images not only are degraded but also lose contrast and color fidelity. Recently, three-dimensional (3D) image technology has been developed intensively. Investment in cutting-edge image production and services, such as Google glasses, Google cardboard, virtual reality, hand-held devices, and wearable systems, has also increased greatly.

However, most recently developed systems are not suitable for outdoor use. One of the reasons is that the produced stereo images are associated with poor image contrast, which causes image features to be less distinctive and confuses stereo matching. In particular, stereo images can be used for creating or enhancing the illusion of depth by stereopsis; this is notably demonstrated by human binocular vision. Stereoscopic visual information from the left and right eyes is combined in the brain to create the perception of 3D depth. Thus, it is necessary to restore images when dealing with outdoor stereo images.

Despite the high demand for 3D outdoor applications or services, few visibility restoration algorithms have been proposed for foggy stereo images. One naive solution is to apply a basic dehazing algorithm to stereo images iteratively. However, most dehazing algorithms are designed to run on a single image. The iterative dehazing of stereo images might cause inconsistent results between the stereo images [9]. In this paper, we study stereo vision and defogging problems jointly, and present an algorithm that simultaneously estimates scene depth and defogs the input stereo images. 
Yu-Chen Wu, Chin-Chen Chang, Der-Lor Way, Zen-Chung Shih; Improved Stereo Image Dehazing Approach, Advances in I mage and Video Processing, Volume 6 No 6, December (2018); pp: 1-7

The proposed approach is based on the observation of depth cues in stereo images. The fog transmission can be directly computed from depth. In this approach, we use the dark channel prior (DCP) technique [7] to improve the transmission results. However, this causes other challenges, including halo effects and false contours, to arise. Thus, we enhance our transmission calculation by combining it with the method of Shiau et al. [12] and with a bilateral filter [16]. The bilateral filter can preserve edges to avoid false contours and the method of Shiau et al. [12] can prevent halo effects. Finally, our approach can avoid inconsistent results between a pair of stereo images based on a depth map. The proposed approach can achieve a better result than a naive method that applies a dehazing algorithm to stereo images iteratively.

The remainder of this paper is organized as follows: In Section 2, we review related works about single image dehazing and stereo image dehazing. Section 3 describes our proposed method and the details of each procedure. Section 4 discusses the results. Section 5 draws conclusions and suggests directions for future research.

\section{Related Works}

Image dehazing is challenging because hazy scenes often suffer from low contract and limited visibility. Therefore, numerous single image dehazing algorithms have been proposed. A typical single image dehazing algorithm attempts to recover a single image from a hazy image. Because a hazy image only conveys ambiguous information about the depicted scene, some single-image dehazing methods require additional data. Kopf et al. [8] used a user registration process to produce depth information and dehaze an image in the context of depth. Some single-image dehazing methods are based on color restoration. Tan's method [13] maximizes local contrast and can obtain airlight, which is the light scattered by particles. Fattal [4] obtained color lines in an image and used them to dehaze the image. Fattal also proposed another method [3] that entails combining the transmission and shading models, and these models were applied to dehaze images. Fattal assumed that, locally, transmission and shading are statistically uncorrelated.

Some other single-image dehazing methods were based on filters. Gibson and Nguyen [5] estimated the amount of fog in an image with a local Wiener filter. They used the theory of Retinex [10], [17] for single image dehazing. Multiscale Retinex with color restoration (MSRCR) can improve the visual rendering of an image even when the lighting conditions are not adequate. Menget et al. [10] introduced a boundary constraint on a transmission function. Tarel and Hautiere [14] introduced a method based on a median filter and combined with an edge preserving filter. He et al. [7] introduced a DCP that exploits the minimum intensity of all color channels in a local window to indicate the level of haziness. Shiau et al. [12] improved the method of He et al. [7] by applying a weighted technique that automatically determines the possible atmospheric lights and mixes these candidates to refine the atmospheric light.

Several dehazing algorithms have been proposed for stereo images. Li et al. [9] presented a method for video defogging and stereo reconstruction. They obtained the similarity between video frames and constructed a depth map according to this similarity. They improved the photo-consistency term to explicitly model the appearance change due to the scattering effect. However, Carr and Hartley [1] proposed an approach to enhance a single image by compensating for fog effects, assuming that the depth and atmospheric conditions are known. Kopf et al. [8] demonstrated that even a rough depth information can also significantly improve the dehazing performance. Moreover, Li et al. [9] concluded that a stereo-image dehazing algorithm based on the constraints of a depth map could obtain a 
favorable result. The constraints of depth maps can counteract some inconsistencies between stereo images.

\section{The Proposed Approach}

The proposed approach initially computes the transmission and disparity. The transmission is computed on the basis of the weighted dark channel method [12]. Simultaneously, the scattering coefficient and atmospheric light vector are computed. Next the fine transmission is calculated based on the cross bilateral filter with transmission and depth maps. Finally, the fine transmission can be transformed to a fine depth map directly or used for dehazing.

\subsection{Stereo Disparity Map}

The depth or disparity map plays a major role because a fine depth map can produce an excellent dehazing result [8]. The depth map of a pair of stereo images can be computed by two processes, namely camera calibration and disparity creation. Camera calibration can be used to undistort the image. In this step we use the method of Zhang [21] for camera calibration. Because we focus on stereo image dehazing, we calibrate the left and right cameras. Therefore, we undistort the left and right images iteratively.

For the disparity map, we use Yang's method [18]. This approach combines nonlocal cost aggregation and a minimum spanning tree. It can produce excellent results in regions with low textures and low complexity. This method can retain most of the shape details. This is especially suitable for a set of hazy stereo images. Yang's method is suitable for parallel programming [19].

\subsection{Dark Channel Weighted Transmission}

The depth or disparity map plays a major role because a fine depth map can produce an excellent dehazing result [8]. The depth map of a pair of stereo images can be computed by two processes, namely camera calibration and disparity creation. Camera calibration can be used to undistort the image. In this step we use the method of Zhang [21] for camera calibration. Because we focus on stereo image dehazing, we calibrate the left and right cameras. Therefore, we undistort the left and right images iteratively.

A transmission is useful when recovering a hazy image. In this step, we recover the transmission through the DCP technique [7]. However, the DCP result is not of sufficient quality. We combine the DCP technique with the weighting function of Shiau et al. [12]. For a hazy image, the observed light is absorbed and scattered by turbid mediums such as atmospheric particles or raindrops. In the context of this observation, a simple atmospheric scattering model [11] can be used to represent the formation of a hazy image. This model is defined as Equations (1) and (2). This model is also used in several dehazing algorithms $[1,3-5,7-10,12-15,17,20]$.

$$
\begin{gathered}
I(x)=J(x) t(x)+A(1-t(x)) \\
t(x)=e^{-\beta d(x)}
\end{gathered}
$$

A guidance hazy image $I$ can be expressed by Equation (1), where $J$ is the clear image, $A$ is the global atmospheric light and $t$ is the transmission. When the atmosphere is homogeneous, the transmission $t$ can be expressed as Equation (2), where $\beta$ is the scattering coefficient and $d(x)$ is the distance from the scene to the camera center. Equation (2) indicates that the scene radiance attenuates exponentially with the depth. Therefore, we can recover a fine depth map if we can recover the transmission.

For each haze-free image, He et al. [7] found that in most of the nonsky patches, at least one color channel has some pixels whose intensities are remarkably low and close to zero. Similarly, the minimum 
Yu-Chen Wu, Chin-Chen Chang, Der-Lor Way, Zen-Chung Shih; Improved Stereo Image Dehazing Approach, Advances in I mage and Video Processing, Volume 6 No 6, December (2018); pp: 1-7

intensity in such a patch is close to zero. Accordingly, He et al. [7] defined such observations as dark channels; these can be formally represented as given in Equation (3):

$$
\min _{y \in \Omega}\left(\min _{c} J_{c}(x)\right)=0
$$

where $c$ indicates the color channel, which is one of red, green, or blue. Hence, $J_{c}$ is the color channel of any arbitrary image $J$ and $\Omega$ is a local patch centered at $x$. A dark channel is the outcome of two minimum operators.

With the dark channel and the assumption that the transmission in a local patch is constant, Equation (1) can be written as Equation (4). We denote this transmission as $\tilde{t}$, and this transmission is constant in the patch.

$$
\min _{y \in \Omega}\left(\min _{c} \frac{I_{c}(x)}{A_{c}}\right)=\min _{y \in \Omega}\left(\min _{c} \frac{J_{c}(x)}{A_{c}}\right) \tilde{t}(x)+(1-\tilde{t}(x))
$$

By substituting Equation (3) into Equation (4), we can obtain a transmission recovery method as expressed in Equation (5).

$$
\tilde{t}(x)=1-\min _{y \in \Omega}\left(\min _{c} \frac{I_{c}(x)}{A_{c}}\right)
$$

The transmission obtained directly from the dark channel is not of adequate quality, especially in the sky region or night image. This is because the estimation of a dark channel is based on patches. Therefore, a distortion problem tends to arise for any dehazing result at any boundary between sky and nonsky regions.

He et al. [7] solved the problem with soft matting. However, that solution is not generally suitable because soft matting is a time-consuming method and might generate inconsistent results for stereo images. Shiau et al. [12] proposed an idea of weighted transmission. The main problem of distortion of the dark channel method is the assumption required for Equation (4). It is assumed that the transmission in a local patch is constant. However, this assumption is inconsistent with the actual result, particularly within any patches between sky and nonsky regions. For the dark channel method, the local minimum or local maximum in such a patch would affect the patch transmission balance. The weighted transmission can avoid such circumstances.

Similarly, we define a difference function in Equation (6). This difference function calculates the ratio of the current pixel dark channel relative to the patch dark channel.

$$
\operatorname{diff}(x)=\frac{\min _{c}\left\{I_{c}(x)\right\}}{\min _{y \in \Omega}\left\{\min _{c}\left\{I_{c}(y)\right\}\right\}}
$$

The weighting function is defined as Equation (7), where $\theta$ is a nonzero positive constant.

$$
W_{\Omega}=\frac{e^{-\theta[\operatorname{diff}(x)-1]}}{\operatorname{diff}(x)}
$$

This weighting function concludes that if there exists a pixel with a significant difference in dark channel relative to the patch, the weight of that pixel must be larger than the weights of other pixels in the same patch. With the aforementioned weighting function, we can rewrite the transmission in Equation (4). Finally, the new transmission is stated as Equation (8).

$$
\tilde{t}(x)=1-\left(\left(1-W_{\Omega}\right) \times \min _{c}\left\{\frac{I^{c}(x)}{A^{c}}\right\}+W_{\Omega} \times \min _{y \in \Omega}\left\{\min _{c}\left\{\frac{I^{c}(x)}{A^{c}}\right\}\right\}\right)
$$




\subsection{Cross Bilateral Filtering}

We use a bilateral filter [2] to jointly combine the depth map and the transmission map. A bilateral filter is a nonlinear, edge-preserving and noise-reducing smoothing filter for images [22]. The intensity value of each pixel is calculated based on a weighting function. The weight is based on a Gaussian distribution; the weight depends on the Euclidean distances between pixels and radiometric differences [16].

The bilateral filter in the proposed approach is used to preserve the edges and restrict the constraint of depth cues. However, a stereo depth map does not work well for large distances [6]. Nevertheless, the transmission contains a depth ordering and a smoothness prior probability. Therefore, in our approach, we use the depth map as a set of radiometric differences to restrict the adjacent pixels' intensities accordingly.

In the proposed approach, the cross bilateral filter is defined as Equation (9).

$$
t_{p}=\frac{1}{W_{p}} \sum_{q \in \Omega_{p}} G_{s}(\|p-q\|) G_{r}\left(\left|D_{p}-D_{q}\right|\right) \tilde{t}_{q}
$$

where $\tilde{t}$ indicates the transmission image created from dark channel weighted transmission, $D$ is the disparity map, $\Omega_{p}$ is the center patch at pixel $p$, and $W_{p}$ is a normalized term, defined by Equation (10).

$$
W_{p}=\sum_{q \in \Omega_{p}} G_{s}(\|p-q\|) G_{r}\left(\left|D_{p}-D_{q}\right|\right)
$$

The spatial similarity $G_{S}$ and the range similarity $G_{r}$ are defined in Equations (11) and (12), where $\sigma^{2}$ and $\varrho^{2}$ are nonzero positive constants.

$$
\begin{aligned}
G_{s}(\|p-q\|) & =\exp \left\{-\frac{\|p-q\|}{2 \sigma^{2}}\right\} \\
G_{r}\left(\left|D_{p}-D_{q}\right|\right) & =\exp \left\{-\frac{\left|D_{p}-D_{q}\right|}{2 \varrho^{2}}\right\}
\end{aligned}
$$

The result $t_{p}$ is the final fine transmission. We can recover the fine depth map directly using Equation (2).

\section{Results}

The proposed approach was run on a PC with a $3.33 \mathrm{GHz}$ Core $^{\mathrm{TM}}$ i7 CPU and $8.0 \mathrm{~GB}$ of memory. The patch size $\Omega$ was used in the dark channel weighted transmission and cross bilateral filter. For relatively small patches, the dehazed images were oversaturated. However, for relatively large patches, the halo effects were stronger along the edges between the sky and nonsky regions.

We used UNITY to simulate fog effects. Figures 1-3 illustrate the results of the fog simulation with an exponential function. For the method of $\mathrm{He}$ et al. [7], the dehazed images appeared overly saturated. For the method of Shiau et al. [12], the colors of distant objects seemed unnatural.
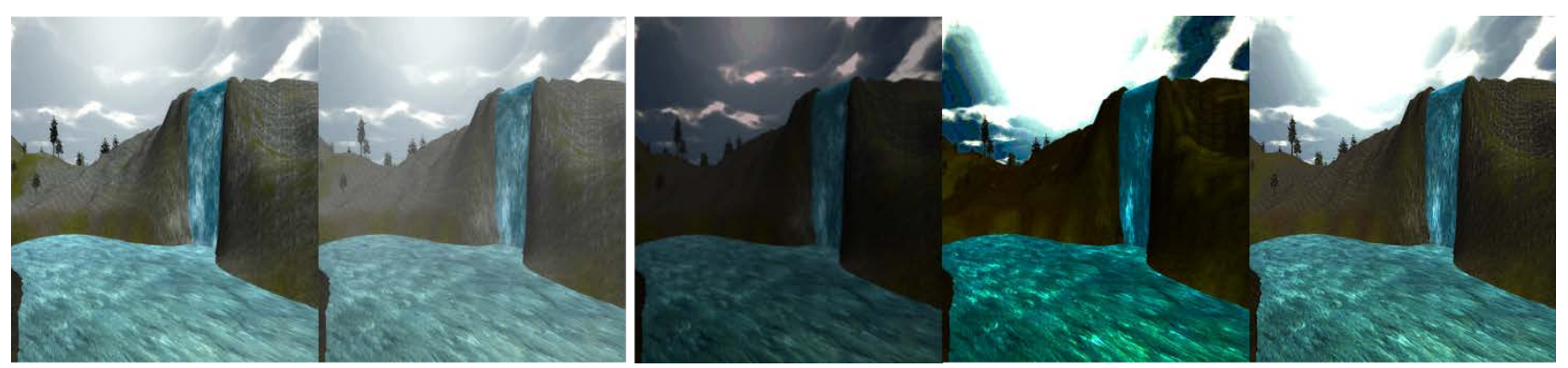

Figure 1. Waterfall: (a) original image, (b) haze image, (c) image dehazed by the method of He et al. [7], (d) image dehazed by the method of Shiau et al. [12], and (e) image dehazed by our approach. 
Yu-Chen Wu, Chin-Chen Chang, Der-Lor Way, Zen-Chung Shih; Improved Stereo Image Dehazing Approach,
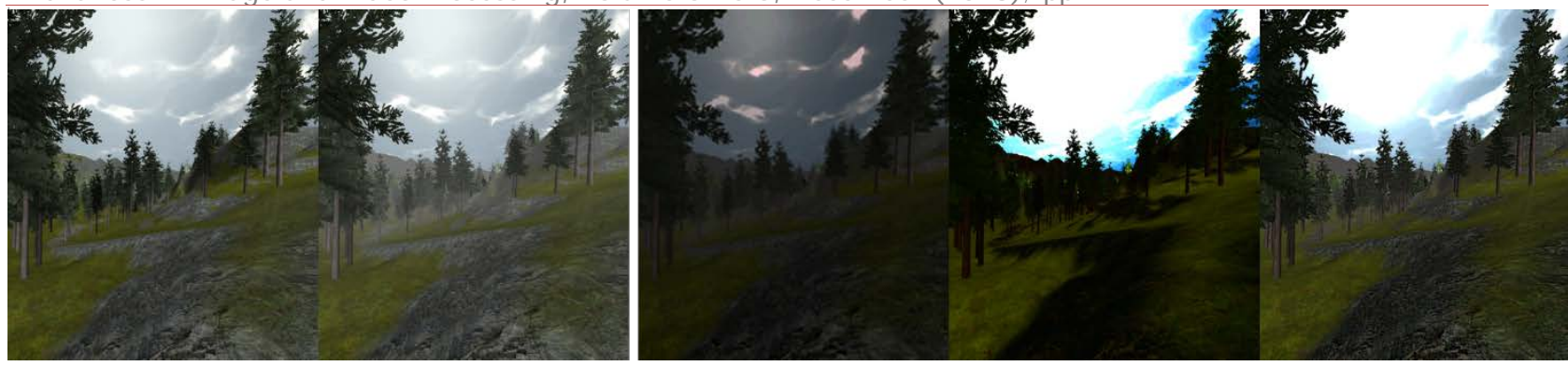

Figure 2. Terrain: (a) original image, (b) haze image, (c) image dehazed by the method of He et al. [7], (d) image dehazed by the method of Shiau et al. [12], and (e) image dehazed by our approach.
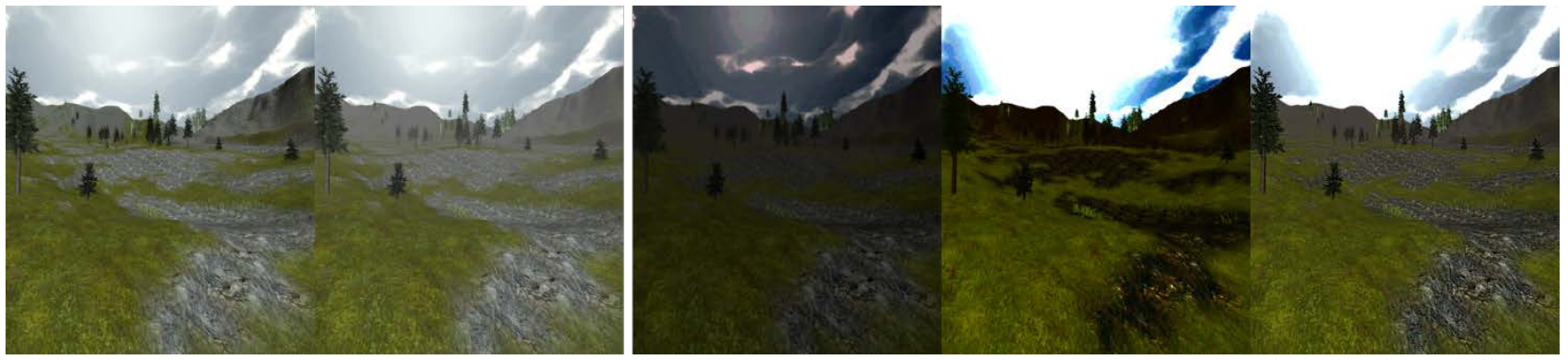

Figure 3. Rock road: (a) original image, (b) haze image, (c) image dehazed by the method of He et al. [7],

(d) image dehazed by the method of Shiau et al. [12], and (e) image dehazed by our approach.

\section{Conclusion}

In this paper, we have proposed a dehazing approach for stereo images. Our approach is based on depth cues and avoids inconsistent results. A cross bilateral filter can constrain the transmission and preserve shape details. The results prove that our approach can obtain high quality dehazed results. The results also demonstrate that it is not proper to dehaze stereo images iteratively. Dehazing methods that incorporate depth information should be considered when dehazing stereo images.

Because our approach requires a disparity map, the quality of the disparity map influences the quality of our approach. The proposed approach may fail for objects with similar colors. We will address this problem for future research.

\section{REFERENCES}

[1] P. Carr, R. Hartley, "Improved Single Image Dehazing Using Geometry", Digital Image Computing: Techniques and Applications (DICTA), 103-110, 2009.

[2] H. Cho, H. Lee, H. Kang, S. Lee, "Bilateral Texture Filtering", ACM Transactions on Graphics (TOG), 33(4), 1281-1288, 2014.

[3] R. Fattal, "Single Image Dehazing", ACM Transactions on Graphics (TOG), 27(3), 7-23, 2008.

[4] R. Fattal, “Dehazing Using Color-Lines”, ACM Transactions on Graphics (TOG), 34(1), 1-14, 2014.

[5] K. Gibson, T. Nguyen, "Fast Single Image Fog Removal Using the Adaptive Wiener Filter", Proceedings of the International Conference on Image Processing (ICIP), 714-718, 2013.

[6] R.I. Hartley, A. Zisserman, Multiple View Geometry in Computer Vision, Cambridge: Cambridge University Press, 2004. 
[7] K. He, J. Sun, X. Tang, "Single Image Haze Removal Using Dark Channel Prior", IEEE Transactions on Pattern Analysis and Machine Intelligence (TPAMI), 33(12), 2341-2353, 2011.

[8] J. Kopf, B. Neubert, B. Chen, M. Cohen, D. Cohen-Or, O. Deussen, M. Uyttendaele, D. Lischinski, “Deep Photo: Model-Based Photograph Enhancement and Viewing", ACM SIGGRAPH Asia, 2008.

[9] Z. Li, P. Tan, R.T. Tan, D. Zou, "Simultaneous Video Defogging and Stereo Reconstruction", Computer Vision and Pattern Recognition (CVPR), 4988-4997, 2015.

[10] G. Meng, Y. Wang, J. Duan, X. Xiang, C. Pan, “Efficient Image Dehazing with Boundary Constraint and Contextual Regularization", International Conference on Computer Vision (ICCV), 617-624, 2013.

[11] W.E.K. Middleton, Vision Through the Atmosphere, Toronto: University of Toronto Press, 1952.

[12] Y.H. Shiau, P.Y. Chen, H.Y. Yang, C.H. Chen, S.S. Wang, "Weighted Haze Removal Method with Halo Prevention", Journal of Visual Communication and Image Representation, 25(2), 445-453, 2014.

[13] R.T. Tan, "Visibility in Bad Weather from A Single Image", Computer Vision and Pattern Recognition (CVPR), 1-8, 2008.

[14] J.P. Tarel, H. Hautiere, "Fast Visibility Restoration from a Single Color or Gray Level Image", International Conference on Computer Vision (ICCV), 2201-2208, 2009.

[15] J.P. Tarel, H. Hautiere, "Improved Visibility of Road Scene Images under Heterogeneous Fog", Proceedings of IEEE Intelligent Vehicle Symposium (IV), 478-485. 2010.

[16] C. Tomasi, R. Manduchi, "Bilateral Filtering For Gray and Color Images", International Conference on Computer Vision (ICCV), 839-846, 1998.

[17] J.B. Wang, N. He, K. Lu, "A New Single Image Dehazing Method with MSRCR Algorithm", Proceedings of the International Conference on Internet Multimedia Computing and Service (ICIMCS), 2015.

[18] Q.X. Yang, “A Non-Local Cost Aggregation Method For Stereo Matching”, Computer Vision and Pattern Recognition (CVPR), 1402-1409, 2012.

[19] Q.X. Yang, N. Ahuja, K.H. Tan, “Constant Time Median and Bilateral Filtering”, International Journal of Computer Vision, 112(3), 307-318, 2015.

[20] Y.C. Wu, C.C. Chang, D.L. Way, Z.C. Shih, "A Novel Dehazing Approach for Stereo Images," in Proceedings of the International Workshop on Advanced Image Technology 2017 (IWAIT 2017), Penang, Malaysia, January 2017.

[21] Z. Zhang, "A Flexible New Technique for Camera Calibration", IEEE Transactions on Pattern Analysis and Machine Intelligence (TPAMI), 22(11), 1330-1334, 2000. 